\title{
Can we Avoid Unfavourable Clinical Herb-Drug Interactions?
}

\author{
Xiao-Wu Chen ${ }^{1 *}$ and Shu-Feng Zhou ${ }^{2 *}$ \\ ${ }^{1}$ Department of General Surgery, the First People's Hospital of Shunde, Southern Medical University, Shunde, Guangdong, 528300, China \\ ${ }^{2}$ Department of Pharmaceutical Sciences, College of Pharmacy, University of South Florida, Tampa, Florida, 33612, USA
}

Herbal medicines are traditionally used by all ethnic groups in folk medicine in a hope of promoting health and managing various diseases such as pains, cold, inflammation, heart diseases, and diabetes, although the mechanism of action is generally unclear and clinical efficacy and safety data are lacking [1]. The 2007 National Health Interview Survey including 23,393 adults aged 18 years or older and 9,417 children aged 17 years and under revealed that $17.7 \%$ of American used natural products mainly including herbs and other supplements. Herbal medicines are often used in combination with conventional drugs, and this may give rise to the potential of unfavorable herb-drug interactions. To date, a number of clinically important herb-drug interactions have been reported [2-6]. The question is how to minimize the occurrence of harmful clinical herb-drug interactions.

\section{What and Why Herbal Medicines and Prescribed Drugs Interact?}

Common herbal medicines that interact with drugs include St John's wort, ginkgo, ginger, ginseng, milk thistle, kava, and garlic [2-6]. However, well documented herb-drug interactions are sparse and many of the observed herb-drug interactions are based on case and case series reports. In many cases, herb-drug interactions may alter drug levels in the body and/or cause adverse drug reactions which may be moderate or fatal. For example, St John's wort significantly reduced the blood concentrations of cyclosporine, midazolam, tacrolimus, amitriptyline, digoxin, indinavir, warfarin, phenprocoumon and theophylline [6]. Common drugs that interact with herbal medicines include warfarin, midazolam, digoxin, amitriptyline, indinavir, cyclosporine, tacrolimus and irinotecan [2]. Herbal medicines may interact with drugs at the intestine, liver, kidneys, and targets of action. Importantly, many of these drugs have very narrow therapeutic indices. Most of them are substrates for cytochrome P450s (CYPs) and/or P-glycoprotein (P-gp/ MDR1).

The underlying mechanisms for most reported herb-drug interactions are not fully understood, and pharmacokinetic and/or pharmacodynamic mechanisms are implicated in many of these interactions [7]. In particular, enzyme induction and inhibition may play an important role in the occurrence of some herb-drug interactions. For example, hyperforin present in St John's wort can activate the pregnane X receptor (PXR/NR1I2) and activation of PXR can lead to increased expression of a panel of genes encoding CYP3A4 and 2B6 and P-gp [6].

\section{Can we Avoid Unfavourable Herb-Drug Interactions?}

The interaction of herbal medicines with prescribed drugs is a significant safety concern, especially for drugs with narrow therapeutic indices (e.g. warfarin and digoxin). In many cases, patients think that herbal medicines are natural products and, thus, are certainly safe. They are not willing to reveal the usage of herbal medicines to clinicians, thus there is little knowledge of who are taking these products and for what indications. As such, herb-drug interactions are highly likely to be significantly under-reported and underestimated, and probably occur more frequently than drug-drug interactions [8]. Because the pharmacokinetics and/or pharmacodynamics of the drug may be altered by combination with herbal remedies, severe and perhaps even life-threatening adverse reactions may occur in clinical practice. Due to the clinical significance of herbal interactions with conventional drugs, it is important to identify clinically important herb-drug interactions and the information is useful for providing warning and proper professional advices to patients in clinical practice or consumers of herbal medicines [9].

Harmful herb-drug interaction may be avoided and reduced by timely warning of the potential risks to patients [10]. However, prediction of clinical herb-drug interaction risks appears to be difficult given that herbal medicines comprise a number of active components that may contribute to herb-drug interactions. A number of drug- and patient-related factors such as extra-hepatic metabolism, auto-induction, active transport in the liver, entero-hepatic circulation, disease status, renal and hepatic functions, and genetic polymorphisms can affect the outcome of herb-drug interactions [3,4].

The dramatic rise in the use of herbal medicine worldwide means that many more patients on conventional medicines are being exposed to herbal medicines. Thus, timely identification of drugs capable of interacting with herbal medicines is important to remind clinicians and drug scientists [9]. Existing knowledge advises us that many herbal medicines should not be taken at the same time with many other drugs that have a narrow therapeutic index. Strategies can be adopted to minimize the risks of herb-drug interactions in drug development.

To date, only a very small proportion of currently available drugs have been investigated for their potential interaction with a small number of herbal medicines such as St John's wort and ginkgo, in humans. Thus, further well-designed randomized clinical studies are certainly required to gain new knowledge of herb-drug interactions when there is strong evidence from preclinical studies. The critical examination of interactions between herbal medicines and drugs requires the ability to accurately determine not only the presence of altered metabolism and transport but also the ability to quantitate the extent of the interaction and clinical consequences in humans.

Since herb-drug combinations are often encountered in clinical

${ }^{*}$ Corresponding authors: Xiao-Wu Chen, MD, Professor, President, Department of General Surgery, the First People's Hospital of Shunde, Southern Medical University, Shunde, Guangdong, 528300, China, Tel: 867572231 8555; Fax: 867572222 3899; E-mail: drchenxiaowu@163.com

Shu-Feng Zhou, MD, PhD, Professor, Associate Dean, Department of Pharmaceutical Sciences, College of Phamacy, University of South Florida, 12901 Bruce B. Downs Boulevard, Tampa, Florida 33612, USA, Tel: 813974 6276; Fax: 813974 9885, E-mail: szhou@health.usf.edu

Received May 23, 2012; Accepted May 23, 2012; Published May 26, 2012

Citation: Chen XW, Zhou SF (2012) Can we Avoid Unfavourable Clinical HerbDrug Interactions? Adv Pharmacoepidem Drug Safety 1:e105. doi:10.4172/21671052.1000e105

Copyright: (C) 2012 Chen XW, et al. This is an open-access article distributed under the terms of the Creative Commons Attribution License, which permits unrestricted use, distribution, and reproduction in any medium, provided the original author and source are credited. 
Citation: Chen XW, Zhou SF (2012) Can we Avoid Unfavourable Clinical Herb-Drug Interactions? Adv Pharmacoepidem Drug Safety 1:e105. doi:10.4172/2167-1052.1000e105

Page 2 of 2

practice and herb-drug interactions have important clinical and toxicological implications, a proper design of drugs that have minimal potential for herbal interaction has important implications in drug development [9]. If the drugs have to be used in combination with herbal medicines, in some instances rational use of such drugs becomes necessary, including the use of a safe drug combination regimen, dose adjustment, and discontinuation of therapy when toxic herb-drug interactions occur. When herbal medicines are combined with drugs with narrow therapeutic indices, monitoring of plasma drug concentrations and close observing of potential adverse effects are always needed. If these drugs have to be used in combination with the herbs, dose adjustment may be needed and discontinuation of therapy is necessary when toxic drug-herb interactions occur. Both patients and clinicians should be educated on the clinical significance of herb-drug interactions.

\section{References}

1. Cordell GA, Colvard MD (2012) Natural products and traditional medicine: turning on a paradigm. J Nat Prod 75: 514-525.
2. Chen XW, Serag ES, Sneed KB, Liang J, Chew H, et al. (2011) Clinical herbal interactions with conventional drugs: from molecules to maladies. Curr Med Chem 18: 4836-4850.

3. Hu Z, Yang X, Ho PC, Chan SY, Heng PW, et al. (2005) Herb-drug interactions: a literature review. Drugs 65:1239-1282.

4. Kennedy DA, Seely D (2010) Clinically based evidence of drug-herb interactions: a systematic review. Expert Opin Drug Saf 9: 79-124.

5. Fugh-Berman A (2000) Herb-drug interactions. Lancet 355: 134-138.

6. He SM, Sneed KB, Chen XW, Cao C, Zhou SF (2012) Herb-drug interactions and mechanistic and clinical considerations. Curr Drug Metab 13: 640-651.

7. Shi S, Klotz U (2012) Drug interactions with herbal medicines. Clin Pharmacokinet. 51: 77-104.

8. Fasinu PS, Bouic PJ, Rosenkranz B (2012) An overview of the evidence and mechanisms of herb-drug interactions. Front Pharmacol. 3: 69.

9. Yang XX, Hu ZP, Duan W, Zhu YZ, Zhou SF (2006) Drug-herb interactions: eliminating toxicity with hard drug design. Curr Pharm Des. 12: 4649-4664.

10. Zhou S, Huang M, Xu A, Yang H, Duan W, et al. (2005) Prediction of herb-drug metabolic interactions: a simulation study. Phytother Res 19: 464-471. 